\title{
Cluster meanfield approximation for the optical response of weakly doped semiconductor quantum wells
}

\author{
Franz X. Bronold ${ }^{1,2}$ \\ ${ }^{1}$ Institut für Theoretische Physik, Otto-von-Guericke-Universität Magdeburg, D-39016 Magdeburg, Germany \\ ${ }^{2}$ Physikalisches Institut, Universität Bayreuth, D-95440 Bayreuth, Germany
}

(Dated: February 7, 2020)

\begin{abstract}
The calculation of the optical properties of doped semiconductor quantum wells is an intricate many-body problem because of the dynamical response of the excess carriers to the photogenerated valence band hole. At low densities, however, where the main effect of the dynamical response is the formation of trions, a simple cluster meanfield approximation can be effectively employed to calculate the optical susceptibility.
\end{abstract}

\section{INTRODUCTION}

The interaction of an optically generated exciton with excess carriers in the conduction band has been the subject of extensive experimental and theoretical investigations. Especially the high density regime attracted much attention and is qualitatively understood [1, 2, 3] : Dynamical screening and Pauli blocking prohibit the formation of bound $e h[4]$ states, the exciton is therefore unstable and its spectral weight distributed among eh scattering states. Residual correlations between the valence band (VB) hole and conduction band (CB) electrons are still important, however, and, depending on the $\mathrm{CB}$ electron to VB hole mass ratio, give rise to a more or less pronounced Fermi edge singularity [5], for instance, in optical absorption spectra.

With the seminal experiment of Kheng et al. [6], which showed that in a weakly n-doped semiconductor quantum well (QW), photogenerated $e h$ pairs give rise to trions, i.e., bound eeh states comprising two $\mathrm{CB}$ electrons and one VB hole, the center of interested shifted to low densities. As a result of numerous theoretical and experimental studies $17,8,19,10,11,12,13,14,15,16,17,18,19,20,121,22$, 23], the importance of eeh states in the low density regime is now unambiguously established and a qualitative understanding of the low density regime is also starting to emerge.

Both the Fermi edge singularity and the trions are consequences of the dynamical response of the $\mathrm{CB}$ electrons to the sudden appearance of the photogenerated VB hole. A typical lowest order process is depicted in Fig. 11. The photogenerated VB hole scatters from one momentum state to another and simultaneously excites a virtual $e \bar{e}$ pair (recall our notation [4]) to compensate for the momentum transfer. At low densities, the Coulomb interaction is strong enough to correlate the VB hole with two CB electrons (the photoexcited CB electron and the CB electron from the $e \bar{e}$ pair) and a (negatively charged) trion appears. At higher $\mathrm{CB}$ electron densities, an arbitrary number of ee pairs is excited, giving rise to, among others, the screening of the Coulomb interaction. At some density, the Coulomb interaction is then too weak to support trions and/or excitons, but the residual correlations might produce a Fermi edge singularity.

The close connection between trion formation at low densities and the appearance of the Fermi edge singularity at high densities has been verified experimentaly [20, 21, 22] but, so far, a unified theoretical description of the full density dependence of the optical susceptibility, based, e.g., on the qualitative considerations of the previous paragraph, is still missing. To construct such a theory is a rather formidable task. Here, we focus therefore on the low density regime, where the cluster meanfield approximation (CMFA) 24] can be effectively used to calculate the optical susceptibility. 12] In contrast to early theoretical studies of trion states [7, 8, 9, 10, 11], the CMFA describes trion and exciton states simultaneously. Moreover, it explicitly accounts for a (low) density of excess carriers. [In this sense it is closely related to the density matrix method of Esser et al. 14] and the exciton-electron T-matrix model of Suris et al. 15].] The high density regime, however, cannot be systematically reached, despite the earlier optimistic assessment of the author. 

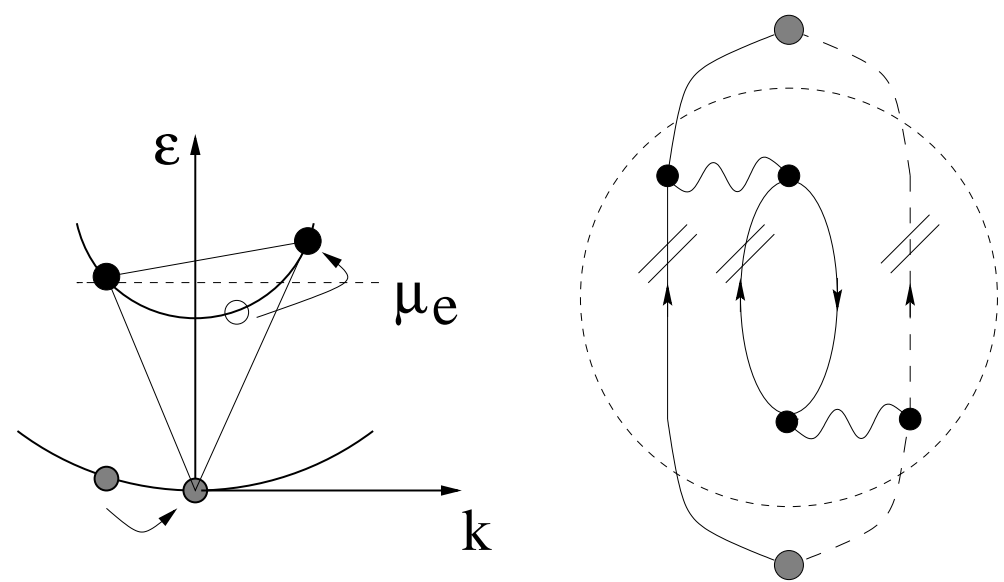

FIG. 1: The left panel illustrates a typical dynamical response of the CB electrons to the photogenerated VB hole in an idealized n-doped semiconductor ( $\mu_{e}$ is the chemical potential for the CB electrons). The right panel shows the corresponding Feynman diagram. The dashed forward running line denotes a VB hole, whereas the forward and backward running solid lines depict, respectively, a CB electron and CB hole. Multiple scattering, indicated by the thin stripes attached to the participating lines, correlates the intermediate eeh cluster to a trion.

\section{CLUSTER MEANFIELD APPROXIMATION (CMFA)}

\section{A. Optical susceptibility}

We consider for simplicity an idealized strictly two-dimensional quantum well with two isotropic parabolic bands. With an appropriate choice of single particle states, our formalism can be of course also applied to more realistic QW models, which include, e.g., valence band mixing and finite band off-sets. We assume a single hole in the VB and a low concentration $n$ of electrons in the CB. The charge carriers are coupled through the Coulomb interaction and the total Hamiltonian is given by

$$
\begin{aligned}
H & =H_{0}+V \\
& =\sum_{\vec{k} \sigma}\left[\epsilon_{c}(k) a_{\vec{k} \sigma}^{\dagger} a_{\vec{k} \sigma}+\epsilon_{v}(k) b_{\vec{k} \sigma}^{\dagger} b_{\vec{k} \sigma}\right]+\frac{1}{2} \sum_{\vec{k} \vec{p} \vec{q} \tau} v(q)\left[a_{\vec{k}+\vec{q} \sigma}^{\dagger} a_{\vec{p}-\vec{q} \tau}^{\dagger} a_{\vec{p} \tau} a_{\vec{k} \sigma}-2 a_{\vec{k}+\vec{q} \sigma}^{\dagger} b_{\vec{p}-\vec{q} \tau}^{\dagger} b_{\vec{p} \tau} a_{\vec{k} \sigma}\right],
\end{aligned}
$$

where $\epsilon_{c}(k)=E_{g}+k^{2} / 2 m_{e}$ and $\epsilon_{v}(k)=k^{2} / 2 m_{h}$ are the CB and VB dispersions $(\hbar=1)$, respectively, and $V(q)=4 \pi e^{2} / \epsilon_{0} q$ is the Coulomb interaction in standard notation.

If the momentum dependence of the dipole matrix element $r_{\gamma}$ is ignored, linear response theory gives for the optical susceptibility ['1' stands for $\vec{k}_{1}$ ]

$$
\chi(\omega)=2\left|r_{\gamma}\right|^{2} \sum_{12}\left\langle\left\langle b_{-1} a_{1} ; a_{2}^{\dagger} b_{-2}^{\dagger}\right\rangle\right\rangle_{\omega}
$$

where $\left\langle\left\langle b_{-1} a_{1} ; a_{2}^{\dagger} b_{-2}^{\dagger}\right\rangle\right\rangle_{\omega}$ denotes the Fourier transform of the two-time correlation function

$$
\left\langle\left\langle\left(b_{-1} a_{1}\right)(t) ;\left(a_{2}^{\dagger} b_{-2}^{\dagger}\right)(0)\right\rangle\right\rangle=-i \Theta(t)\left\langle\left[\left(b_{-1} a_{1}\right)(t),\left(a_{2}^{\dagger} b_{-2}^{\dagger}\right)(0)\right]\right\rangle .
$$

Here, the thermodynamic expectation value is defined by $\langle\ldots\rangle=Z^{-1} \operatorname{Tr}\left(e^{-\beta \mathcal{H}} \ldots\right)$, with $Z=\operatorname{Tr} e^{-\beta \mathcal{H}}$, and $\mathcal{H}=H-\mu_{e} N_{e}$. The imaginary part of $\chi(\omega)$ determines, apart from a constant factor, the optical absorption $I_{a b s}(\omega) \sim-\operatorname{Im} \chi(\omega)$. The overall factor of two in front of the sum on the rhs of Eq. (2) comes from the spin.

\section{B. Dyson-type equations}

The CMFA, originally developed to attack the nuclear many-body problem, rearranges the equations of motion for two-time correlation functions into an hierarchy of Dyson-type equations. It is thus closely 
related to the Mori memory function method, although the mathematical details are quite different. Using CMFA techniques [24], we can derive a formally exact Dyson-type equation for the eh pair propagator $P(12 ; \omega)=\left\langle\left\langle b_{-1} a_{1} ; a_{2}^{\dagger} b_{-2}^{\dagger}\right\rangle\right\rangle_{\omega}$. Keeping in mind that the spin configurations of the various correlation functions are fixed, because of the spin-independence of the Coulomb scattering, we suppress the spin variables and write:

$$
[\omega+i \eta-Z(1)] P(12 ; \omega)=N(1)\left\{\delta_{12}+\sum_{3}\left[M^{s t}(13)+\delta M(13 ; \omega)\right] P(32 ; \omega),\right.
$$

with

$$
\begin{aligned}
M^{s t}(12) & =N^{-1}(1) N^{-1}(2)\left\langle\left[\left[b_{-1} a_{1}, V\right], a_{2}^{\dagger} b_{-2}^{\dagger}\right]\right\rangle \\
\delta M(12 ; \omega) & =N^{-1}(1) N^{-1}(2)\left\langle\left\langle\left[b_{-1} a_{1}, V\right] ;\left[V, a_{2}^{\dagger} b_{-2}^{\dagger}\right]\right\rangle\right\rangle_{\omega} \\
N(1) & =\left\langle\left[b_{-1} a_{1}, a_{2}^{\dagger} b_{-2}^{\dagger}\right]\right\rangle \\
Z(1) & =\epsilon_{c}(1)+\epsilon_{v}(-1)
\end{aligned}
$$

Applying the CMFA technique to the correlation function defining $\delta M(12 ; \omega)$, and so on, we obtain an hierarchy of Dyson-type equations for correlation functions with an increasing number of particles. Clearly this set of equations has to be truncated and it is the truncation which restricts the CMFA to low CB electron densities.

We now briefly recall the truncation procedure adopted in Ref. [12]. The vanishing VB hole concentration implies $\left\langle b_{1}^{\dagger} b_{1}\right\rangle=0$, i.e., $N(1)=1-\left\langle a_{1}^{\dagger} a_{1}\right\rangle$. By assumption, the CB electron density is also small, i.e., $N(1) \simeq 1$. Thus, in leading order in the CB electron density, we ignore Pauli blocking. In the low density regime, we also neglect screening and single-particle selfenergy corrections and Eq. (4) reduces to

$$
P(12 ; \omega)=P^{(0)}(12 ; \omega)+\sum_{34} P^{(0)}(13 ; \omega)\left[M^{s t}(34)+\delta M(34 ; \omega)\right] P(42 ; \omega),
$$

with a bare $e h$ pair propagator, $P^{(0)}(12 ; \omega)=\delta_{12}[\omega+i \eta-Z(1)]^{-1}$, and a static part of the $e h$ pair selfenergy $M^{s t}(45)=-v(4-5)$. The dynamical response of the CB electrons, i.e., the creation of virtual $e \bar{e}$ pairs, is encoded in the dynamical (resonating) part of the $e h$ pair selfenergy, which, in the low density regime, becomes

$$
\begin{aligned}
\delta M(12 ; \omega) & =\sum_{4567} v(5) v(7)(R(1,4,4+5,5-1 \mid 2,6,6+7,7-2 ; \omega) \\
& -R(1-5,4,4-5,-1 \mid 2,6,6+7,7-2 ; \omega) \\
& -R(1,4,4+5,5-1 \mid 2-7,6,6-7,-2 ; \omega) \\
& +R(1-5,4,4-5,-1 \mid 2-7,6,6-7,-2 ; \omega)) .
\end{aligned}
$$

We defined an eightpoint eeēh function $R(1235 \mid 5678 ; \omega)=\left\langle\left\langle b_{4} a_{3}^{\dagger} a_{2} a_{1} ; a_{5}^{\dagger} a_{6}^{\dagger} a_{7} b_{8}^{\dagger}\right\rangle\right\rangle_{\omega}$, for which,as indicated above, we again derive a Dyson-type equation, symbolically written as $R(\omega)=R^{(0)}(\omega)+R^{(0)}(\omega)\left[K^{s t}+\right.$ $\delta K(\omega)] R(\omega)$. [Assuming that the spin configuration of the photogenerated eh pair is $(\uparrow \downarrow)$, the spin configuration of the eee h cluster defining $R$ is $(\uparrow \sigma \sigma \downarrow) ; \sigma$ is then summed over in Eq. (10).] In the dilute limit, we need $\delta M(12 ; \omega)$ only to $o(n)$. Using an $\bar{e}$-line expansion for $\delta M(12 ; \omega)$, we can show (i) that the term $\delta K(\omega)$ gives rise to $o\left(n^{2}\right)$ contributions to $\delta M(12 ; \omega)$ and is thus negligible at low densities, and (ii) that, in leading order in the CB electron density, the eightpoint eeēh function appearing in Eq. (10) in fact factorizes into

$$
R(1234 \mid 5678 ; \omega)=\delta_{37} f_{e}(3) G_{3}\left(12 \mid 56 ; \omega+\epsilon_{c}(3)\right)
$$

with $f_{e}(3)=<a_{3}^{\dagger} a_{3}>$ and a sixpoint eeh function $G_{5}(12 \mid 34 ; \omega)=\left\langle\left\langle b_{5-1-2} a_{2} a_{1} ; a_{3}^{\dagger} a_{4}^{\dagger} b_{5-3-4}^{\dagger}\right\rangle\right\rangle_{\omega}$, which satisfies

$$
\left[\omega+i \eta-Z_{5}(12)\right] G_{5}(12 \mid 34 ; \omega)=I(12 \mid 34)+\sum_{67} \mathcal{V}_{5}(12 \mid 67) G_{5}(67 \mid 34 ; \omega)
$$

with

$$
\mathcal{V}_{5}(12 \mid 67)=v(7-2) \delta_{6,1+2-7}-v(1-6) \delta_{2,7}-v(2-7) \delta_{6,1}
$$


and

$$
Z_{5}(12)=\epsilon_{c}(1)+\epsilon_{c}(2)+\epsilon_{v}(5-1-2) .
$$

Thus, at low densities, the hierarchy of Dyson-type equations can be truncated at the level of a sixpoint eeh function and, in leading order in the CB electron density, the optically generated eh pair is only coupled to an eeh cluster. The groundstate of the eeh cluster has antiparallel CB electron spin. Focusing on the eeh groundstate, we fix therefore the spin configuration of the eeh cluster defining $G$ to ( $\uparrow \downarrow$ ). For this spin configuration, $I(12 \mid 34)=\delta_{13} \delta_{24}$, and the spin eventually leads to the overall factor of two in Eq. (2) for the optical susceptibility.

Using the spectral representation for the sixpoint eeh function,

$$
G_{5}(12 \mid 34 ; \omega)=\sum_{n} \frac{\Psi_{5 n}(12) \Psi_{5 n}^{*}(34)}{\omega+i \eta-\Omega_{n}(5)},
$$

we finally obtain for the dynamic part of the eh pair selfenergy

$$
\delta M(34 ; \omega)=\sum_{5 n} f_{e}(5) \frac{\Xi_{5 n}(3) \Xi_{5 n}^{*}(4)}{\omega+i \eta+\epsilon_{c}(5)-\Omega_{n}(5)},
$$

where all multiple scattering events within the eeh cluster are now encoded in a vertex function

$$
\Xi_{5 n}(3)=\sum_{7} v(5-7)\left[\Psi_{5 n}(37)-\Psi_{5 n}(7,3+5-7)\right],
$$

given in terms of (normalized and complete) momentum-space eeh wavefunctions $\Psi_{5 n}(37)$, which satisfy an eeh momentum-space Schrödinger equation:

$$
\left[\Omega_{n}(5)-Z_{5}(12)\right] \Psi_{5 n}(12)-\sum_{34} \mathcal{V}_{5}(12 \mid 34) \Psi_{5 n}(34)=0
$$

Here, 'n' and '5' depict, respectively, internal quantum numbers and the (center-of-mass) momentum of the (propagating) bound eeh cluster.

To calculate $P(12 ; \omega)$ it is advantageous to split Eq. (9) into two coupled integral equations with, respectively, $M^{s t}(=-v)$ and $\delta M$ as kernels:

$$
\begin{aligned}
P^{s t}(12 ; \omega) & =P^{(0)}(12 ; \omega)-\sum_{34} P^{(0)}(13 ; \omega) v(3-4) P^{s t}(42 ; \omega) \\
P(12 ; \omega) & =P^{s t}(12 ; \omega)+\sum_{34} P^{s t}(13 ; \omega) \delta M(34) P(42 ; \omega)
\end{aligned}
$$

The first integral equation is readily solved using again a spectral representation

$$
P^{s t}(12 ; \omega)=\sum_{\nu} \frac{\Phi_{\nu}(1) \Phi_{\nu}^{*}(2)}{\omega+i \eta-E_{\nu}}
$$

where the (normalized and complete) momentum-space exciton wave functions $\Phi_{\nu}(1)$ obey an eh momentum-space Schrödinger equation (Wannier equation)

$$
\left[E_{\nu}-Z(1)\right] \Phi_{\nu}(1)+\sum_{2} v(1-2) \Phi_{\nu}(2)=0
$$

The solutions of this equation are analytically known. 25]

The second integral equation cannot be solved exactly. In order to avoid heavy numerics, we employ for its solution a simple approximation scheme based on the "exciton representation", i.e., on a bilinear expansion in terms of the solutions of Eq. (22). Writing $P(12 ; \omega)=\sum_{\nu, \mu} P_{\nu \mu}(\omega) \Phi_{\nu}(1)^{*} \Phi_{\mu}(2)$, we transform Eq. (20) into an infinite set of algebraic equations for the expansion coefficients,

$$
\left[\omega+i \eta-E_{\nu}\right] P_{\nu \mu}(\omega)=\delta_{\nu \mu}+\sum_{\lambda} \delta M_{\nu \lambda}(\omega) P_{\lambda \mu}(\omega)
$$




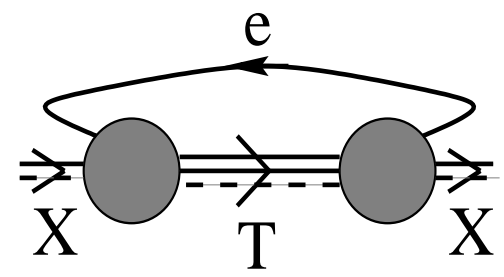

FIG. 2: Graphical illustration (not a Feyman diagram) of the dynamical part of the exciton selfenergy $\delta M_{X}(\omega)$. The full circles denote the exciton-trion coupling constants $u_{5 T}^{X}$ and $\left[u_{5 T}^{X}\right]^{*}$.

where

$$
\delta M_{\nu \lambda}(\omega)=\sum_{5 n} f_{e}(5) \frac{u_{5 n}^{\nu}\left[u_{5 n}^{\lambda}\right]^{*}}{\omega+i \eta+\epsilon_{c}(5)-\Omega_{n}(5)}
$$

and $u_{5 n}^{\nu}=\sum_{1} \Phi_{\nu}^{*}(1) \Xi_{5 n}(1)$. In the vicinity of the exciton resonance, i.e., for energies $\omega$ close to the exciton groundstate energy $E_{X}$, we expect the exciton and trion groundstate to be dominant. Thus, we solve Eq. (23) by employing one-pole approximations: First, we keep only the term corresponding to the exciton ground state $\nu=X$ and neglect the coupling to excited exciton states $\nu \neq X$. Next, assuming that the main modification of the exciton ground state comes from the groundstate of the eeh cluster, i.e., from the trion denoted by $n=T$, we restrict the sum in Eq. (24) to the term $n=T$.

Let us emphasize, the one-pole approximations are by no means mandatory and various improvements are possible. For example, if the exciton binding energy is small, i.e., if the exciton groundstate is not well separated from the first excited state, the first excited state has to be kept. This gives rise to two coupled algebraic equations (two-pole approximation). It is even conceivable to treat all $e h$ scattering states in a similar way, introducing an "effective" excited state, which carries the spectral weight of the whole continuum. Excited trion states can be also taken into account by simply extending the sum in Eq. (24) to the respective states.

The theoretical work by Esser et al. 14] indicates that these refinements are indeed necessary to obtain good quantitative agreement between theory and experiment. For the purpose of demonstration, however, we neglect here scattering states and stick to the simple one-pole approximation described above. The optical susceptibility in the vicinity of the exciton resonance is then given by

$$
\chi(\omega) \simeq \frac{2\left|s_{X}\right|^{2}}{\omega+i \eta-E_{X}-\delta M_{X}(\omega)},
$$

with an exciton selfenergy (see Fig. 2)

$$
\delta M_{X}(\omega)=\sum_{5} f_{e}(5) \frac{\left|u_{5 T}^{X}\right|^{2}}{\omega+i \eta+\epsilon_{c}(5)-\Omega_{T}(5)},
$$

and an exciton oscillator strength $s_{X}=r_{\gamma} \sum_{1} \Phi_{X}(1)$. Note, the one-pole approximation is conceptually close to the T-matrix model considered by Suris et al. in their analysis of absorption and reflection spectra of modulation-doped quantum wells. 15]

To proceed further, we assume that the CB electron density is such that the Fermi function in Eq. (26) can be replaced by a Boltzmann function parametrized by the CB electron density $n$. Measuring energies in units of the $2 \mathrm{D}$ exciton binding energy $4 R(R$ is the $3 \mathrm{D}$ exciton Rydberg) and length in units of the 3D Bohr radius $a_{B}$, we define $\tilde{\chi}(\tilde{\omega}) \equiv \chi\left(4 R \tilde{\omega}+E_{g}\right) / 4 R$, with $\tilde{\omega}=\left[\omega-E_{g}\right] / 4 R$, and rewrite the optical susceptibility into dimensionless form

$$
\tilde{\chi}(\tilde{\omega})=\frac{\left|s_{X}\right|^{2}}{8 R^{2}} \frac{1}{\tilde{\omega}+i \tilde{\eta}+1-\delta \tilde{M}_{X}(\tilde{\omega})} .
$$

Here, $\tilde{\eta}=\eta / 4 R$ and

$$
\begin{aligned}
\delta \tilde{M}_{X}(\tilde{\omega}) & \equiv \delta M_{X}\left(4 R \tilde{\omega}+E_{g}\right) / 4 R \\
& =\tilde{\beta} \frac{M_{T}}{M_{X}}\left(n a_{B}^{2}\right)\left|u_{T}^{X}\right|^{2} I(\tilde{\omega}),
\end{aligned}
$$


where $\tilde{\beta}=4 \beta R, M_{X}$, and $M_{T}$ are, respectively, the thermal energy measured in units of $4 R$, the exciton mass, and the trion mass. The exponential integral,

$$
I(\tilde{\omega})=\int_{0}^{\infty} d y \frac{e^{-\tilde{\beta} \frac{M_{T}}{M_{X}} y}}{\tilde{\omega}+i \tilde{\eta}-\tilde{\epsilon}_{T}+y},
$$

where $\tilde{\epsilon}_{T}=\left[\Omega_{T}(0)-2 E_{g}\right] / 4 R$ is the energy of a trion at rest, i.e., the internal trion energy, which is the binding energy of two $\mathrm{CB}$ electrons to one $\mathrm{VB}$ hole. The dimensionless exciton-trion coupling constant $u_{T}^{X}$ finally is given by an integral involving the wave function of the trion groundstate, the Coulomb interaction, and the wavefunction of the exciton groundstate. For simplicity we neglected the weak dependence of $u_{T}^{X}$ on the (center-of-mass) momentum of the trion. Note, for vanishing CB electron density $n, \delta \tilde{M}_{X}(\tilde{\omega}) \rightarrow 0$ and $\tilde{\chi}(\tilde{\omega})$ describes a single exciton resonance.

To obtain the exciton-trion coupling constant $u_{T}^{X}$ and the internal trion energy $\tilde{\epsilon}_{T}$, we employ the variational technique originally used by Stébé and coworkers [9, 10] to calculate various properties of an isolated trion. For that purpose we transform Eq. (18) to real space (to solve the eeh problem we use atomic units, which are, however, at the end of the calculation, translated into the excitonic units adopted above)

$$
[T+V] \Psi_{T}\left(\vec{r}_{e 1} \vec{r}_{e 2} \vec{r}_{h}\right)=\epsilon_{T} \Psi_{T}\left(\vec{r}_{e 1} \vec{r}_{e 2} \vec{r}_{h}\right),
$$

with kinetic and potential energies $(\hbar=1)$,

$$
\begin{aligned}
T & =-\frac{1}{2 m_{e}}\left(\Delta_{e 1}+\Delta_{e 2}\right)-\frac{1}{2 m_{h}} \Delta_{h}, \\
V & =-\frac{e^{2}}{\epsilon_{0}}\left(\frac{1}{\left|\vec{r}_{e 1}-\vec{r}_{h}\right|}+\frac{1}{\left|\vec{r}_{e 2}-\vec{r}_{h}\right|}-\frac{1}{\left|\vec{r}_{e 1}-\vec{r}_{e 2}\right|}\right),
\end{aligned}
$$

separate away the center-of-mass motion, which is simply a plane wave, and make a variational Hylleraas Ansatz for the internal part of the trion groundstate wavefunction,

$$
\Psi(s, t, u)=\sum_{l n m} c_{l n m}|\operatorname{lnm}\rangle,
$$

where $|\operatorname{lnm}\rangle=e^{-\frac{s}{2}} s^{l} t^{m} u^{n}$ and $u, s$, and $t$ are ellipitical coordinates for the internal motion of the eeh cluster. Note, since the trion groundstate is a singlet, $\Psi(s, t, u)$ has to be an even function in $t$, i.e., $m$ has to be even. The expansion coefficients are determined by a Ritz variational principle, i.e., by minimizing the energy functional $E\left[\vec{c}, \epsilon_{T}\right]=\langle\Psi|T+V| \Psi\rangle /\langle\Psi \mid \Psi\rangle$. The variation yields an eigenvalue problem which is then iteratively solved. The largest eigenvalue is related to $\epsilon_{T}\left(\rightarrow \tilde{\epsilon}_{T}\right)$ and the associated eigenfunction gives the expansion coefficients $c_{l n m}$. For more details we refer the readers to Refs. [9, 10] and [26]. With the Hylleraas variational function, the exciton-trion coupling constant becomes

$$
u_{T}^{X}=\sqrt{\frac{2}{N \pi}} \frac{1}{k(1+\sigma)} \int_{0}^{\infty} d s \int_{0}^{s} d u \int_{0}^{u} d t \frac{2 \pi\left(s^{2}-t^{2}\right) u}{\sqrt{\left(u^{2}-t^{2}\right)\left(s^{2}-u^{2}\right)}} e^{-\frac{s+t}{k(1+\sigma)}}\left[\frac{2}{s-t}-\frac{1}{u}\right] \Psi(s, t, u),
$$

where $k$ is the effective charge of a CB electron, $N$ is the norm of $\Psi$, and $\sigma=m_{e} / m_{h}$.

\section{Some numerical results}

Using a 2D 22-term Hylleraas wavefunction, we calculated in Ref. 12 the optical absorption along the lines explained in the previous subsections. For a mass ratio $\sigma=m_{e} / m_{h}=0.146$, corresponding to GaAs, we found $u_{T}^{X} \simeq 1.0896$ and $\tilde{\epsilon}_{T}=-1.1151$. ( $u_{T}^{X}$ was obtained by Gaussian quadrature.) The binding energy (in units of $4 R$ ) for one electron is therefore $\tilde{W}_{T}=0.1151$ in agreement with the binding energies obtained by other means. 7]

The optical absorption in the vicinity of the exciton resonance is related to the exciton spectral function

$$
D_{X}(\tilde{\omega})=-\operatorname{Im} \tilde{\chi}(\tilde{\omega})
$$

shown in Fig. 3 for $n a_{B}^{2}=0.008$ and $\tilde{\beta}=10$. The overall structure is in qualitative agreement with experiments, despite the simplicity of the model. Below a narrow peak at $\tilde{\omega}=-0.94$, we see a broad 


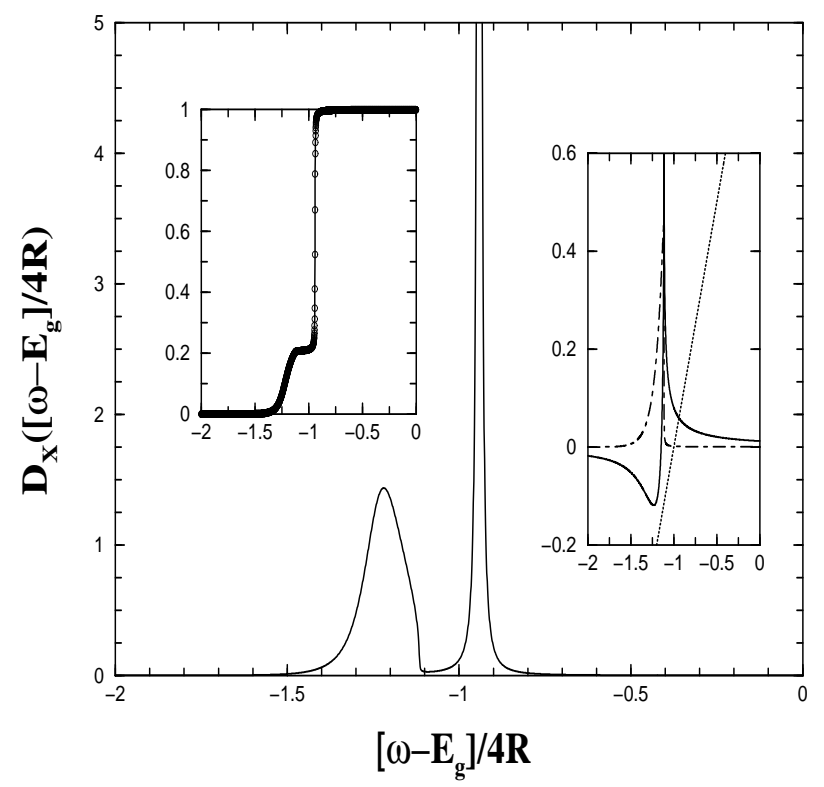

FIG. 3: The spectral function $D_{X}(\tilde{\omega})$ for $n a_{B}^{2}=0.008$ and $4 R \beta=10$. The effective mass ratio $\sigma=0.146$, corresponding to GaAs, and $\tilde{\eta}=0.001$. The left inset depicts the integrated spectral weight $C_{X}(\tilde{\omega})$, whereas the right inset displays $\tilde{\Gamma}_{X}(\tilde{\omega})$ (dot-dashed line) and $\tilde{\Delta}_{X}(\tilde{\omega})$ (solid line). The energy for which $\tilde{\Delta}_{X}(\tilde{\omega})$ crosses $\tilde{\omega}+1$ (dotted line) defines the exciton line at $\tilde{\omega}=-0.94$. From Ref. 12].

absorption band with a sharp high energy edge at $\tilde{\omega}=-1.1151$ and a low energy tail. The narrow peak is the exciton resonance whereas the broad band corresponds to the absorption due to a trion. As shown in Ref. [12], the sharp high energy edge of the absorption band comes from the pole of the exciton selfenergy at $\tilde{\epsilon}_{T}=-1.1151$, i.e., a photon in resonance with the high energy edge creates a trion with momentum zero. The band has a tail at the low energy side because of the recoil of the trion, i.e., the tail comprises trion states with finite momentum. The right inset of Fig. 3 displays the real and imaginary parts of the exciton selfenergy, $\delta \tilde{M}_{X}(\tilde{\omega})=\tilde{\Delta}_{X}(\tilde{\omega})-i \tilde{\Gamma}_{X}(\tilde{\omega})$. As can be seen, trion states with small momenta (high energy edge) are strongly damped. The maximum of the absorption band does therefore not correspond to a trion with zero momentum (as in the case of vanishing CB electron concentration [11]) but to a trion state with a finite momentum. The left inset depicts the integrated spectral weight up to an energy $\tilde{\omega}$ defined in terms of the cumulant $C_{X}(\tilde{\omega})=\int_{-\infty}^{\tilde{\omega}} d \tilde{E} D_{X}(\tilde{E})$. As a consequence of the one-pole approximation, the total spectral weight associated with the trion band and the exciton line adds up to one. If scattering states had been taken into account, they would also carry some spectral weight, i.e., in a complete theory, the combined spectral weight of trion and exciton has to be of course less then one. Our exploratory calculation neglected scattering states, because, at low enough CB densities, in particular, within the range of validity of our theory, scattering states are expected to carry almost no spectral weight. Indeed, the more refined calculations of Esser et al. 14] seem to verify this assessment. The spectral weight, which can be associated with scattering states, is less then $5 \%$ of the spectral weight of the exciton groundstate.

\section{CONCLUSIONS}

We presented a detailed description of the CMFA for the optical response of a weakly n-doped idealized semiconductor QW. The CMFA, tailormade for low CB electron densities, reduces the calculation of the optical susceptibility to the solution of the Schrödinger equations for, respectively, a single $e h$ pair and a single eeh cluster. Excess carriers are only treated as a reservoir for bound state formation, which does not, in leading order in the CB electron density, modify the properties of the eh and eeh states. We derived the CMFA for an idealized QW model, but it is straightforward to generalize the formalism to more realistic models. Furthermore, relaxing the one-pole approximations, which we solely used for illustration, would allow to take exciton and trion continuum states into account. At intermediate-tohigh CB electron density, many-body medium corrections, such as Pauli blocking, screening, and single 
particle selfenergy corrections, substantially modify eh and eeh states. Within the CMFA it is however not clear how to systematically account for these effects.

[1] Haug, H. and Schmitt-Rink, S. (1984), Electron theory of the optical properties of laser-excited semiconductors, Prog. Quant. Electr. 9, 3-100.

[2] Schmitt-Rink, S., Chemla, D. S., and Miller, D. A. B. (1989), Linear and nonlinear optical properties of semiconductor quantum wells, Adv. Phys. 38, 89-188.

[3] Zimmermann, R. (1988), Many-Particle Theory of Highly Excited Semiconductors, Teubner Texte für Physik, Bd. 18, Teubner Verlagsgesellschaft, Leibzig.

[4] $e, \bar{e}$, and $h$ denote, respectively, CB electrons, CB holes, and VB holes; a trion, e.g., is therefore a eeh cluster.

[5] For a recent review see, e.g., Brum, J. A. and Hawrylak, P. (1997), Fermi egde singularity in the optical properties of two-dimensional electron gas, Comments Cond. Mat. Phys. 18, 135-161.

[6] Kheng, K., Cox, R. T., Merle d'Aubigné, Y., Bassani, F., Saminadayar, K., and Tatarenko, S. (1993), Observation of negatively charged excitons $X^{-}$in semiconductor quantum wells, Phys. Rev. Lett. 71, 17521755.

[7] Usukura, J., Suzuki, Y., Varga, K. (1999), Stability of two- and three-dimensional excitonic complexes, Phys. Rev. B 59, 5652-5661.

[8] Ruan, W. J., Chan, K. S., Ho, H. P., Zhang, R. Q., Pun, E. Y. P. (1999), Hyperspherical approach for charged excitons in quantum wells, Phys. Rev. B 60, 5714-5720.

[9] Stébé, B. and Ainane, A. (1989), Ground-state energy and optical absorption of excitonic trions in twodimensional semiconductors, Superlattices and Microstructures 5, 545-548.

[10] Stébé, B., Munschy, G., Stauffer, L., Dujardin, F., and Murat, J. (1997), Excitonic trion $X^{-}$in semiconductor quantum wells, Phys. Rev. B 56, 12454-12461.

[11] Stébé, B., Feddi, E., Ainane, A., and Dujardin, F. (1998), Optical and magneto-optical absorption of negatively charged excitons in three- and two-dimensional semiconductors, Phys. Rev. B 58, 9926-9932.

[12] Bronold, F. X. (2000), Optical absorption of a weakly n-doped semiconductor quantum well, Phys. Rev. B 61, 12620-12623.

[13] Esser, A., Runge, E., Zimmermann, R., and Langbein, W. (2000), Photoluminescence and radiative lifetime of trions in GaAs quantum wells, Phys. Rev. B 62, 8232-8239.

[14] Esser, A., Zimmermann, R., and Runge, E. (2001), Theory of trion spectra in semiconductor nanostructures, phys. stat. sol. (b) 227, 317-330.

[15] Suris, R. A., Kochereshko, V. P., Astakhov, G. V., Yakovlev, D. R., Ossau, W., Nürnberger, J., Faschinger, W., Landwehr, G., Wojtowicz, T., Karczewski, G., and Kossut, J. (2001), Excitons and Trions modified by interaction with a two-dimensional electron gas, phys. stat. sol. (b) 227, 343-353.

[16] Lovisa, S., Cox, R. T., Magnea, N., and Saminadayar, K. (1997), Filling-factor dependence of the negativelycharged-exciton absorption in a CdTe quantum well, Phys. Rev. B 56, R12787-R12790.

[17] Siviniant, J., Scalbert, D., Kavokin, A. V., Coquillat, D., Lascaray, J.-P. (1999), Chemical equilibrium between excitons, electrons, and negatively charged excitons in semiconductor quantum wells, Phys. Rev. B 59, 1602-1604.

[18] Eytan, G., Yayon, Y., Rappaport, M. , Shtrikman, H., and Bar-Joseph, I. (1998), Near-Field spectroscopy of gated electron gas: A direct evidence for electron localization, Phys. Rev. Lett. 81, 1666-1669.

[19] Manassen, A., Cohen, E., Arza Ron, Linder, E., and Pfeiffer, L. N. (1996), Exciton and trion spectral line shape in the presence of an electron gas in GaAs/AlAs quantum wells, Phys. Rev. B 54, 10609-10613.

[20] Shields, A. J., Pepper, M., Ritchie, D. A., and Simmons, M. Y. (1995), Influence of excess electrons and magnetic fields on Mott-Wannier excitons in GaAs quantum wells, Adv. Phys. 44, 47-72.

[21] Brown, S. A., Young, Jeff F., Brum, J. A., Hawrylak, P., and Wasilewski, Z. (1996), Evolution of the interband absorption threshold with the density of a two-dimensional electron-gas, Phys. Rev. B 54, R11082-R11085.

[22] Yusa, G., Shtrikman, H., and Bar-Joseph, I. (2000), Onset of exciton absorption in modulation-doped GaAs quantum wells, Phys. Rev. B 62, 15390-15393.

[23] For a recent comprehensive overview see, e.g., Zimmermann, R. (Ed.) (2001), Proceedings of the Miniworkshop on Trion Physics, phys. stat. sol. (b) 227.

[24] See, e.g., Dukelsky, J., Röpke, G., and Schuck, P. (1998) Generalized Brückner-Hartree-Fock theory and self-consistent RPA, Nucl. Phys. A 628, 17-40.

[25] Haug, H. and Koch, S. W. (1990), Quantum Theory of the Optical and Electronic Properties of Semiconductors, World Scientific, Singapore.

[26] Bethe, H. A. and Salpeter, E. E. (1957), Quantum Mechanics of one- and two-electron atoms, Springer Verlag, Berlin. 